\title{
Outcomes and patterns of radiation associated brain image changes after proton therapy for head and neck skull base cancers
}

\author{
Grete May Engeseth ${ }^{\mathrm{a}, \mathrm{b}, \mathrm{c}, \mathrm{d}}$, Sonja Stieb ${ }^{\mathrm{a}}$, Abdallah Sherif Radwan Mohamed ${ }^{\mathrm{a}}$, Renjie He $^{\mathrm{a}}$,

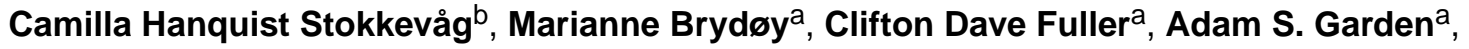 \\ David I. Rosenthal ${ }^{\mathrm{a}}$, Jack Phan ${ }^{\mathrm{a}}$, William H. Morrison ${ }^{\mathrm{a}}$, Jay P. Reddy ${ }^{\mathrm{a}}$, Richard Wua ${ }^{\mathrm{a}}$, \\ Xiaodong Zhanga ${ }^{a}$, Steven Jay Frank ${ }^{a}$, Gary Brandon Gunn ${ }^{a},{ }^{*}$ \\ aDepartment of Radiation Oncology, University of Texas MD Anderson Cancer Center, Houston, \\ USA; \\ ${ }^{b}$ Department of Oncology and Medical Physics, Haukeland University Hospital, Bergen, Norway; \\ 'Department of Clinical Science, University of Bergen, Norway; \\ dDepartment of Physics and Technology, University of Bergen, Norway
}

\section{Abstract}

Background and purpose: To characterize patterns and outcomes of brain MR image changes after proton therapy (PT) for skull base head and neck cancer (HNC).

Material and methods: Post-treatment MRIs $\geq 6$ months were reviewed for radiationassociated image changes (RAIC) in 127 patients. All patients had received at least a point dose of $40 \mathrm{~Gy}(\mathrm{RBE})$ to the brain. The MRIs were rigidly registered to planning CTs and RAIC lesions were contoured both on T1 weighted (post-contrast) and T2 weighted sequences, and dosevolume parameters extracted. Probability of RAIC was calculated using multistate survival analysis. Univariate/multivariate analyses were performed using Cox Regression. Recursive partitioning analysis was used to investigate dose-volume correlates of RAIC development.

Results: $17.3 \%$ developed RAIC. All RAIC events were asymptomatic and occurred in the temporal lobe (14), frontal lobe (6) and cerebellum (2). The median volume of the contrast enhanced RAIC lesion was $0.5 \mathrm{cc}$ at their maximum size. The RAIC resolved or improved in $45.5 \%$ of the patients and were stable or progressed in $36.4 \%$. The 3-year actuarial rate of developing RAIC was $14.3 \%$. RAIC was observed in $63 \%$ of patients when $V_{67} \mathrm{~Gy}(\mathrm{RBE})$ of the brain $\searrow 0.17 \mathrm{cc}$.

\footnotetext{
"Corresponding author at: Department of Radiation Oncology, University of Texas MD Anderson Cancer Center, 1840 Old Spanish Trail, 77054 Houston, TX, USA., GBGunn@ mdanderson.org (G. Brandon Gunn).

Conflict of Interest

None.

Appendix A. Supplementary data

Supplementary data to this article can be found online at https://doi.org/10.1016/j.radonc.2020.07.008.
} 
Conclusion: Small RAIC lesions after PT occurred in $17.3 \%$ of the patients; the majority in nasopharyngeal or sinonasal cancer. The estimated dose-volume correlations confirm the importance of minimizing focal high doses to brain when achievable.

Radiation therapy for head neck cancers (HNCs) at the skull base can result in high doses of radiation delivered to the brain, potentially leading to radiation-associated image changes (RAIC) [1]. Often the diagnosis of RAIC is based solely on radiographic findings on posttreatment MRIs obtained for routine cancer surveillance purposes. For HNCs, information on RAIC stems largely from studies in patients with nasopharyngeal cancers (NPC) treated with Intensity Modulated Radiation Therapy (IMRT), with reported incidences of temporal lobe RAIC ranging between $2 \%$ and $14 \%$ [2-12]. From patients treated proton therapy (PT) a few mixed cohort studies including selected HNCs report incidences of brain RAIC of 11$31 \%$ [13-16]. In studies evaluating the dose-response relationship for RAIC development, constraints have been suggested for several dose-volume parameters as well as for the Equivalent Uniform Dose (EUD) (Table 1), whereas the recently published international consensus guideline for NPC radiotherapy recommend to aim for a temporal lobe $D_{0.03 \mathrm{cc}} \leq$ 65 Gy and $D_{0.03 \mathrm{cc}} \leq 70$ Gy for T1-T2 and T3-T4 tumors, respectively [17].

Owing to the proximity to Central Nervous System (CNS) critical structures, HNCs with tumor at the skull base or with intracranial extension are often selected for treatment with PT to improve or maintain target volume coverage while respecting normal tissues tolerance and reducing the toxicities and symptoms associated with the greater integral dose with IMRT. However, studies on the development of RAIC after PT for HNCs are limited. Thus, as part of a broader effort to better define the role and value of PT for HNC and to provide PTspecific dose recommendations to guide clinicians and the PT community, the specific aims of this study were to:

Characterize the incidence and patterns of RAIC after PT for skull base HNCs Identify candidate clinical and dose-volume parameters associated with RAIC Propose practical dose constraints to minimize the risk of RAIC

\section{Methods and materials}

\section{Patient cohort}

Adult patients treated with PT for HNC at University of Texas MD Anderson Cancer Center were eligible for participation in two consecutive Institutional Review Board-approved prospective studies (ClinicalTrials.org identifiers: NCT 00991094 and NCT 01627093). Eligibility included: (1) Anatomic tumor location at the base of skull, including primary tumors of the nasopharynx, sinonasal, periorbital, parotid, and skin, (2) A point dose of at least 40 Gy to the brain (relative biological effectiveness $=1.1$ ), and (3) one or more followup MRI $ð 6$ months after completion of PT. Patients with prior radiation therapy to the region or who had received photon-based treatment as part of therapy were excluded. 


\section{Treatment}

All PT plans were based on non-contrast CT images and generated in the Eclipse treatment planning system (TPS) (Varian Medical Systems, Palo Alto, CA, USA) using either Intensity Modulated Proton Therapy (IMPT), Passive Scattered Proton Therapy (PSPT) or a combination of IMPT/PSPT. For dose prescription a proton relative biological effectiveness (RBE) value of 1.1 was used as recommended by the ICRU [18]. Dose (prescribed to CTV) and fractionation regimens were generally $60 \mathrm{~Gy}(\mathrm{RBE})$ in 30 fractions or 63-66 Gy(RBE) in 30-33 fractions in the postoperative PT setting or 66-70 Gy(RBE) in 33-35 fractions in the definitive PT setting. Induction chemotherapy regimens used were typically 3 cycles with taxane and platinum or taxane, platinum and fluorouracil (TPF), and concurrent chemotherapy was usually platinum-based.

\section{Follow-up and diagnosis of RAIC}

Follow-up MRIs took place regularly at 8-12 weeks after therapy completion and then every 3 months in the first year, every 4 months in the second year, every 6 months until 5 years, and annually thereafter. Defined as RAIC were (i) contrast enhanced lesions on T1 weighted (T1w) MRIs located in previously irradiated brain tissue and/or (ii) cysts or white matter lesions manifested as high signal intensity on the T2 weighted (T2w) sequences [19]. All RAICs were initially diagnosed by a radiologist during routine disease surveillance and were, for this analysis, identified through review of all MRI reports, and retrospectively reassessed by two board certified radiation oncologists (GBG and SJF). No additional RAIC to those already diagnosed in the MRI reports was discovered. The clinical grading of RAIC was performed retrospectively by chart review according to CTCAE v4.03 [20].

\section{Extraction of clinical and treatment related data}

Patient, tumor and treatment characteristics were retrieved from the patients' medical records (Epic Systems Corporation, Verona, WI) and the TPS. The MRIs were rigid registered to the initial treatment planning $\mathrm{CT}$ and qualitatively evaluated through visual inspection and further manually corrected if deemed necessary. To detect the extent of the RAIC, gadolinium contrast enhanced lesions on T1w sequences (T1w lesions) and hyperintensities including visible edema from $\mathrm{T} 2 \mathrm{w}$ sequences ( $\mathrm{T} 2 \mathrm{w}$ lesions) were contoured as individual structures and propagated to the planning CT. dose-volume histograms (DVHs) for all patients were exported using bin size of $0.1 \mathrm{~Gy}(\mathrm{RBE})$ from where candidate dose volume parameters for the brain tissue were retrieved, including: the maximum dose $\left(\mathrm{D}_{\max }\right)$, the dose delivered to $0.5 \mathrm{cc}\left(\mathrm{D}_{0.5 \mathrm{cc}}\right)$ and $1 \mathrm{cc}\left(\mathrm{D}_{1 \mathrm{cc}}\right)$ to $5 \mathrm{cc}\left(\mathrm{D}_{5 \mathrm{cc}}\right)$ of the brain in $1 \mathrm{cc}$ steps, as well as the volume of brain receiving 40-70 Gy(RBE) in $1 \mathrm{~Gy}(\mathrm{RBE})$ steps $\left(\mathrm{V}_{40 \mathrm{~Gy}(\mathrm{RBE})}\right.$ to $\left(\mathrm{V}_{70 \mathrm{~Gy}(\mathrm{RBE})}\right)$. The selection of candidate variables were based on (a) previously identified dose-volume parameters judged as important in predicting RAIC and (b) applicability to clinical practice and plan optimization and evaluation. In addition, for each patient the $\mathrm{T} 1 \mathrm{w}$ and $\mathrm{T} 2 \mathrm{w}$ lesion volumes with the corresponding minimum dose $\left(D_{\min }\right)$, the mean dose ( $\left.D_{\text {mean }}\right)$ and the $D_{\max }$ were extracted for the first MRI and the MRI with the largest volume of RAIC ("worst" MRI). 


\section{Statistical analysis}

The follow-up time was calculated as the time between the last PT fraction and the patient's last follow-up MRI. The RAIC latency time was calculated as the time between the last PT fraction and first MRI with RAIC. Time to resolution from RAIC was calculated from date of the first MRI with RAIC and the date of the first MRI where lesion had resolved.

In a course where the event of interest can transient between different states, multistate analysis can provide better accuracy of events reporting. As RAIC resolution is common in a considerable proportion of patients, multistate analysis was used to estimate the probability of RAIC over time [21]. For the current analysis the event of interest was RAIC, and data were censored if patients were alive and RAIC free at last available follow-up MRI. Bootstrapping $(n=1000)$ was used to estimate the $95 \%$ confidence intervals $[22,23]$.

Comparisons between patients with and without RAIC were performed using the Mann Whitney U test, the Chi Square and Fishers exact test. All tests were two-sided with a significance level of 0.05 . Correlations between the clinical and dosimetric predictor variables were calculated using Spearman's rank correlation and Pearson's correlation coefficient. Univariate and multivariate analysis of the association between RAIC and candidate predictors were performed using Cox Proportional Hazards Regression Analysis [24,25]. Covariates with $p$ value $<0.2$ from the univariate analysis were considered for inclusion in the multivariate analysis. However, since DVH parameters are highly correlated, the dose volume variables with the lowest significance level from variables where the correlation coefficient were $>0.8$ were chosen for the analysis.

To identify important $V_{\mathrm{D}}$ parameters, recursive partitioning analysis (RPA) was applied. The chosen endpoint was RAIC three years post-treatment; dose-volume statistics from patients with three years follow-up time or more were included in the analysis. RPA was performed using bootstrapping $(n=1000)$ with number of observations in the terminal node constrained to $10 \%$ of the dataset and splitting of nodes based on the Gini index [26,27]. Statistical analyses were performed in SPSS version 24 (IBM SPSS Statistics for Windows, Version 24.0. Armonk, NY: IBM Corp, US) and R version 3.6 (R Foundation for Statistical Computing, Vienna, Austria).

\section{Results}

One-hundred and ninety-three patients treated from 12/2010 through 06/2018 met the inclusion criteria. Of these, 66 were excluded due to prior photon treatment to the same region. The remaining 127 patients formed the cohort.

Patient, tumor and treatment characteristics are displayed in Table 2. Eighty-five patients $(66.9 \%)$ were treated exclusively with IMPT, 15 patients (11.8\%) exclusively with PSPT and 27 patients (21.3\%) with a combination of PSPT and IMPT. Thirty eight patients (29.9\%) had one adaption of the treatment plan and 6 patients $(4.7 \%)$ had two. Twenty patients $(15.7 \%)$ received both induction and concurrent chemotherapy. 
Median follow-up time was 29 months (range: 6-97). Twenty-two patients (17.3\%) developed RAIC with a median latency of 24 months (9-37); all but one RAIC had developed within three years after PT. Median follow-up time after diagnosis of RAIC was 14 months (0-70). All patients with RAIC were asymptomatic at initial diagnoses and subsequent follow-up visits. However, due to progression of the image changes on the follow-up MRIs, two patients were treated with pentoxifylline and vitamin E (one of whom also received dexamethasone), and a third patient was treated with bevacizumab [28]. A decision to initiate treatment suggests that the lesions were considered to be clinically severe enough to warrant medical intervention, therefore, 3 patients were rated to have Grade 3 and 19 patients Grade 1 CNS necrosis according to CTCAE 4.03 (Supplementary material Table 1). At last available MRI the lesions had resolved in six patients, progressed in four, regressed in four, were stable in four and four patients had yet to have a follow-up MRI. A case example of the observed RAIC evolution including overview of evolution timeline can be found in Supplementary Material Figs. 1 and 2. Overall, the estimated probability of RAIC at two, three and five years was $8.7 \%$ (95\% CI: 3.8\%, 13.7\%), $14.3 \%$ (95\% CI: $8.2 \%$, $20.4 \%$ ), and $12.7 \%$ (95\% CI: 6.9\%, 18.5\%), respectively (Supplementary Fig. 3).

Tumor and treatment characteristics for patients with RAIC including dose-volume summary statistics for the contoured lesions are provided in Supplementary Materials (Table 2-4). Of patients diagnosed with RAIC, $68.2 \%$ patients had been treated for T4 or unresectable disease, and $63.6 \%$ had intracranial tumor extension. RAIC was located in the temporal lobe (14 patients), frontal lobe (six patients) and in the cerebellum (two patients). The majority of RAIC developed in patients with NPC or sinonasal cancers (77.3\%); all (eight) RAIC occurred in the temporal lobe for the NPCs and for the patients with sinonasal cancer RAIC was found both in the temporal lobe (four) and the frontal lobe (five) (Supplementary Material Fig. 4). Two of the contrast-enhanced T1w lesions were inside and seven were outside the CTV, the remaining 13 were overlapping with the CTV. At first MRI $92 \%$ of the T1w contrast-enhanced lesions were $1 \mathrm{cc}$ or less, with a median volume of $0.3 \mathrm{cc}$ (punctuate to $1.1 \mathrm{cc}$ ); on the MRIs where the RAIC was at its largest size, $77 \%$ of the T1w lesions were less than $1 \mathrm{cc}$ with a median volume of $0.5 \mathrm{cc}(0.1-3.1 \mathrm{cc})$. The $\mathrm{T} 1 \mathrm{w}$ lesion doses were highest in lesions located in the temporal lobe and for lesions inside the CTV (Fig. 1).

Statistical significant differences by RAIC group were only found for prescribed dose and brain dose-volume parameters (Tables $2-3) . V_{40} \mathrm{~Gy}(\mathrm{RBE})-V_{70 \mathrm{~Gy}(\mathrm{RBE})}$ for the brain were included in the RPA. The result showed that $63.1 \%$ of the patients with $V_{67} \mathrm{~Gy}(\mathrm{RBE}) \geq 0.17 \mathrm{cc}$ developed RAIC (Fig. 2/Supplementary Material Table 5). Based on the result from RPA, we included $V_{67} \mathrm{~Gy}(\mathrm{RBE}) \geq 0.17 \mathrm{cc}$ in the univariate cox regression analysis. From this analysis prescribed dose, $D_{\max }, D_{1-5 \mathrm{cc}}, V_{70 \text { (GyRBE) }}$ and $V_{67 \mathrm{~Gy}(\mathrm{RBE})} \geq 0.17 \mathrm{cc}$ were significantly associated with the development of RAIC. In the multivariate analysis; $V_{67 \mathrm{~Gy}(\mathrm{RBE})} \geq 0.17 \mathrm{cc}$ was the only statistically significant predictive factor (HR: $7.57,95 \%$ CI: $1.48,38.76, p=0.015$ ) (Table 4).

\section{Discussion}

In the current study, we characterized the patterns and clinical outcomes of RAIC after PT in a relatively large and heterogeneous cohort of patients with HNCs at the skull base. The 
crude incidence rate of RAIC in our study was $17.3 \%$, but given the transient nature of observed lesions, the estimated probability of RAIC at 2, 3, and 5-years was 9\%, 14\%, and $13 \%$, respectively.

In contrast to reported elsewhere, the patients in our study where asymptomatic. There are several possible explanations for our findings in contradistinction to other series. Both higher doses and larger RAIC volumes have been suggested to have an impact on the development of clinical symptoms $[1,28,29]$. In the current study the median $\mathrm{T} 1 \mathrm{w}$ and $\mathrm{T} 2 \mathrm{w}$ lesion volumes were $0.5 \mathrm{cc}$ and $2.9 \mathrm{cc}$ at its worst, whereas lesion volumes reported in other studies were considerable larger. Zhou et al. [12] found mean T1w and T2w lesion volumes of $4.2 \mathrm{cc}$ and $21.7 \mathrm{cc}$, and $30 \%$ clinical symptoms. Su et al. [6] reported mean T1w and T2w lesion volumes of $7.7 \mathrm{cc}$ and $21.2 \mathrm{cc}$ and $22 \%$ clinical symptoms. McDonald et al. [13] found significant difference in the mean and maximum lesion doses between Grade 1 vs. Grade 2 lesions. In the McDonald study the $D_{\text {mean }}$ and $D_{\max }$ for symptomatic contrast enhancing lesions were 73.0 $\mathrm{Gy}(\mathrm{RBE})$ and $82.9 \mathrm{~Gy}(\mathrm{RBE})$, respectively, compared to $62.2 \mathrm{~Gy}(\mathrm{RBE})$ and $69.0 \mathrm{~Gy}(\mathrm{RBE})$ for asymptomatic lesions in the current study. Secondly, asymptomatic RAIC could be considered an early sign of impending symptomatic progression, future development of clinical symptoms in our cohort cannot be ruled out. Thirdly, as no formal assessment of neuro-cognitive function was routinely performed, it is possible that the clinical impact of the lesions and corresponding edema is underestimated.

In RPA all possible combinations of thresholds among the $V_{\mathrm{D}}$ parameters were evaluated and showed that $63 \%$ of the patients with $V_{67} \mathrm{~Gy}$ (RBE) $\searrow 0.17 \mathrm{cc}$ developed RAIC; the $V_{67 \mathrm{~Gy}(\mathrm{RBE})}$ therefore provides practical and useful reference to support clinical decision making, particularly in difficult cases where higher dose to the brain is unavoidable and where the risk of developing RAIC is high. Our result is in good agreement with the recently published international consensus guideline for NPC, that recommend avoiding $V_{65}$ Gy $\geq$ $0.03 \mathrm{cc}$ and $\mathrm{V}_{70 \text { Gy }} \geq 0.03 \mathrm{cc}$ for $\mathrm{T} 1-\mathrm{T} 2$ and $\mathrm{T} 3-\mathrm{T} 4$ tumors, respectively [17]. The result agrees reasonable well with the proposed dose constraint from McDonald et al. [13], who identified of $V_{70 \mathrm{~Gy}(\mathrm{RBE})}<1.7 \mathrm{cc}$ for $15 \%$ probability of RAIC using Generalized Estimating Equation modelling of dose response relationship with RAIC in 66 patients treated with PT.

Niyazi et al. [14] used DVH reduction methods when developing a mixture NTCP model for patients with intracranial- and extracranial tumors treated with PT. An a-value of 9 fitted their data best, and they estimated an $\mathrm{EUD}_{9}=39.5 \mathrm{~Gy}$ for $50 \%$ probability of symptomatic CNS necrosis. It is not possible to directly compare this to our result, however, an a-value of 1 equals the mean dose, while with increasing a-values the high dose area of the DVH curve are more weighted and the calculated EUD gets closer to the near-maximum dose.

An important goal of the current study was to provide a comprehensive overview over the incidence of RAIC in HNC treated with proton therapy. HNC are a heterogeneous patient group with varying dose to the brain, depending on both dose prescription and tumor location; a brain dose threshold as one of the inclusion criteria was therefore considered appropriate. Previous studies have identified $\mathrm{V}_{40 \mathrm{~Gy}}$ as one predictor of RAIC and in order to ensure identification of all RAIC events in the cohort, we selected $D_{\max } \geq 40 \mathrm{~Gy}(\mathrm{RBE})$ as the minimum cut-off point. 
Although all MRI reports and images in the current study were retrospectively reviewed and diagnoses verified, inherent limitations of retrospective review apply. Other limitations in our study exist, including a relatively short follow-up time, as RAIC can develop at a later time point or existing lesions could potentially progress. The MRIs in our study were acquired for standard post treatment surveillance purposes, and T2 flair sequences were not routinely available, thus standard $\mathrm{T} 2 \mathrm{w}$ sequences were used for contouring $\mathrm{T} 2 \mathrm{w}$ edema signal, which can be less accurate than T2 flair. Differences in scanner types and image acquisition protocols inherently influence image quality, sensitivity and specificity and could influence the interpretation of the images. However, as the MRIs were only used for detection and contouring of the brain image changes the uncertainty should therefore be small with simple sequences as $\mathrm{T} 1 \mathrm{w}$ and $\mathrm{T} 2 \mathrm{w}$. Further, since the lesions were very small, accuracy of the calculation of the lesion doses may be influenced by uncertainties in the image registration procedure. Finally, our material included a variety of tumor sites treated with both PSPT and IMPT. PSPT generally has a larger irradiated volume, whereas IMPT is conformal, but more sensitive to uncertainties. Although PT technique was not significantly correlated with RAIC development, we cannot rule out that it may influence our results. In addition, the study could also have limited power to define dose-volume constraints since our cohort was too small to split into a training and a validation set for RPA. Therefore, our results should be externally validated.

We investigated RAIC in a heterogeneous cohort of skull base HNC and found that $17.3 \%$ developed RAIC, the majority of these occurred in nasopharyngeal or sinonasal cancers. The lesions were small and asymptomatic, resolved in $27 \%$ of the patients and progressed in $18 \%$ of the patients. The estimated dose-volume correlations identified in our study were in agreement with previous studies and confirm the importance of minimizing focal high doses to brain when achievable.

\section{Supplementary Material}

Refer to Web version on PubMed Central for supplementary material.

\section{Acknowledgement of grants or other financial support}

Engeseth is funded by Trond Mohn Foundation. S Stieb is funded by the Swiss Cancer League (BIL

KLS-4300-08-2017). Stokkevåg is funded by Kreftforeningen. Fuller received funding and salary support related to this project from the National Institutes of Health $(\mathrm{NIH})$, including: the National Institute for Dental and Craniofacial Research Establishing Outcome Measures Award (1R01DE025248/R56DE025248) and an Academic Industrial Partnership Grant (R01DE028290). Dr. Fuller received funding and salary support unrelated to this during the project from: National Science Foundation (NSF), Division of Mathematical Sciences, Joint NIH/NSF Initiative on Quantitative Approaches to Biomedical Big Data (QuBBD) Grant (NSF 1557679); a National Institute of Biomedical Imaging and Bioengineering (NIBIB) Research Education Programs for Residents and Clinical Fellows Grant (R25EB025787-01); the NIH Big Data to Knowledge (BD2K) Program of the National Cancer Institute (NCI) Early Stage Development of Technologies in Biomedical Computing, Informatics, and Big Data Science Award (1R01CA214825); NCI Early Phase Clinical Trials in Imaging and Image-Guided Interventions Program (1R01CA218148); an NIH/NCI Cancer Center Support Grant (CCSG) Pilot Research Program Award from the UT MD Anderson CCSG Radiation Oncology and Cancer Imaging Program (P30CA016672) and an NIH/NCI Head and Neck Specialized Programs of Research Excellence (SPORE) Developmental Research Program Award (P50 CA097007). Dr. Fuller has received direct industry grant support, honoraria, and travel funding from Elekta AB. S.J. Dr. Dr. Frank reports personal fees from Varian, grants and personal fees from C4 Imaging, grants from Eli Lilly, grants from Elekta, grants and personal fees from Hitachi, other from Breakthrough Chronic Care, personal fees from Boston Scientific, and personal fees from National Comprehensive Cancer Center (NCCN). Dr. Gunn reports philanthropic donation from the Family of Paul W. Beach. 


\section{References}

[1]. Lawrence YR, Li XA, el Naqa I, Hahn CA, Marks LB, Merchant TE, et al. Radiation dose-volume effects in the brain. Int J Radiat Oncol Biol Phys 2010;76(3 Suppl): S20-7. [PubMed: 20171513]

[2]. Feng M, Huang Y, Fan X, Xu P, Lang J, Wang D. Prognostic variables for temporal lobe injury after intensity modulated-radiotherapy of nasopharyngeal carcinoma. Cancer Med 2018;7:55764. [PubMed: 29473319]

[3]. Huang J, Kong FF, Oei RW, Zhai RP, Hu CS, Ying HM. Dosimetric predictors of temporal lobe injury after intensity-modulated radiotherapy for T4 nasopharyngeal carcinoma: a competing risk study. Radiat Oncol 2019;14:31. [PubMed: 30736809]

[4]. Kong C, Zhu XZ, Lee TF, Feng PB, Xu JH, Qian PD, et al. LASSO-based NTCP model for radiation-induced temporal lobe injury developing after intensity-modulated radiotherapy of nasopharyngeal carcinoma. Sci Rep 2016;6:26378. [PubMed: 27210263]

[5]. Su SF, Huang SM, Han F, Huang Y, Chen CY, Xiao WW, et al. Analysis of dosimetric factors associated with temporal lobe necrosis (TLN) in patients with nasopharyngeal carcinoma (NPC) after intensity modulated radiotherapy. Radiat Oncol 2013;8:17. [PubMed: 23336282]

[6]. Su SF, Huang Y, Xiao WW, Huang SM, Han F, Xie CM, et al. Clinical and dosimetric characteristics of temporal lobe injury following intensity modulated radiotherapy of nasopharyngeal carcinoma. Radiother Oncol 2012;104:312-6. [PubMed: 22857858]

[7]. Sun Y, Zhou GQ, Qi ZY, Zhang L, Huang SM, Liu LZ, et al. Radiation-induced temporal lobe injury after intensity modulated radiotherapy in nasopharyngeal carcinoma patients: a dosevolume-outcome analysis. BMC Cancer 2013;13:397. [PubMed: 23978128]

[8]. Takiar V, Ma D, Garden AS, Li J, Rosenthal DI, Beadle BM, et al. Disease control and toxicity outcomes for T4 carcinoma of the nasopharynx treated with intensity-modulated radiotherapy. Head Neck 2016;38(Suppl 1):E925-33. [PubMed: 25994561]

[9]. Wang J, Miao Y, Ou X, Wang X, He X, Shen C, et al. Development and validation of a model for temporal lobe necrosis for nasopharyngeal carcinoma patients with intensity modulated radiation therapy. Radiat Oncol 2019;14:42. [PubMed: 30866964]

[10]. Zeng L, Huang SM, Tian YM, Sun XM, Han F, Lu TX, et al. Normal tissue complication probability model for radiation-induced temporal lobe injury after intensity-modulated radiation therapy for nasopharyngeal carcinoma. Radiology 2015;276:243-9. [PubMed: 25658039]

[11]. Zhou GQ, Yu XL, Chen M, Guo R, Lei Y, Sun Y, et al. Radiation-induced temporal lobe injury for nasopharyngeal carcinoma: a comparison of intensity-modulated radiotherapy and conventional two-dimensional radiotherapy. PLoS One 2013;8:e67488. [PubMed: 23874422]

[12]. Zhou X, Ou X, Xu T, Wang X, Shen C, Ding J, et al. Effect of dosimetric factors on occurrence and volume of temporal lobe necrosis following intensity modulated radiation therapy for nasopharyngeal carcinoma: a case-control study. Int J Radiat Oncol Biol Phys 2014;90:261-9. [PubMed: 25066214]

[13]. McDonald MW, Linton OR, Calley CS. Dose-volume relationships associated with temporal lobe radiation necrosis after skull base proton beam therapy. Int J Radiat Oncol Biol Phys 2015;91:261-7. [PubMed: 25636754]

[14]. Niyazi M, Niemierko A, Paganetti H, Sohn M, Schapira E, Goldberg S, et al. Volumetric and actuarial analysis of brain necrosis in proton therapy using a novel mixture cure model. Radiother Oncol 2019.

[15]. Dagan R, Bryant C, Li Z, Yeung D, Justice J, Dzieglewiski P, et al. Outcomes of sinonasal cancer treated with proton therapy. Int J Radiat Oncol Biol Phys 2016;95:377-85. [PubMed: 27084655]

[16]. Pehlivan B, Ares C, Lomax AJ, Stadelmann O, Goitein G, Timmermann B, et al. Temporal lobe toxicity analysis after proton radiation therapy for skull base tumors. Int J Radiat Oncol Biol Phys 2012;83:1432-40. [PubMed: 22349042]

[17]. Lee AW, Ng WT, Pan JJ, Chiang CL, Poh SS, Choi HC, et al. International guideline on dose prioritization and acceptance criteria in radiation therapy planning for nasopharyngeal carcinoma. Int J Radiat Oncol Biol Phys 2019;105:567-80. [PubMed: 31276776] 
[18]. International Commission on Radiation Units and Measurements. Report 78: prescribing, recording and reporting proton-beam therapy. Oxford: International Commission on Radiation Units and Measurements; 2007.

[19]. Shah R, Vattoth S, Jacob R, Manzil FF, O’Malley JP, Borghei P, et al. Radiation necrosis in the brain: imaging features and differentiation from tumor recurrence. Radiographics 2012;32:134359. [PubMed: 22977022]

[20]. NCI Common Terminology Criteria for Adverse Events (CTCAE): National Cancer Institute 2010 [Available from: https://evs.nci.nih.gov/ftp1/CTCAE/CTCAE_4.03/ CTCAE_4.03_2010-06-14_QuickReference_5×7.pdf.

[21]. Wang YX, King AD, Zhou H, Leung SF, Abrigo J, Chan YL, et al. Evolution of radiationinduced brain injury: MR imaging-based study. Radiology 2010;254:210-8. [PubMed: 20019142]

[22]. Aalen OO, Johansen S. An empirical transition matrix for non-homogeneous Markov chains based on censored observations. Scand J Stat 1978;5:141-50.

[23]. Datta S, Satten GA. Validity of the Aalen-Johansen estimators of stage occupation probabilities and Nelson-Aalen estimators of integrated transition hazards for non-Markov models. Stat Prob Lett 2001;55:403-11.

[24]. Therneau T, Grambsch PM. Modeling survival data: extending the Cox model. New York: Springer; 2000.

[25]. Therneau T A package for survival analysis in R. version 2.382015 [Available from: https:// CRAN.R-project.org/package=survival].

[26]. Kuhn M caret: Classification and regression training. R package version 6.0-84 2019 [Available from: https://CRAN.R-project.org/package=caret].

[27]. Therneau T, Atkinson B rpart: Recursive Partitioning and Regression Trees. R package version 4.1-15 2019 [Available from: https://CRAN.R-project.org/package=rpart].

[28]. Rahmathulla G, Marko NF, Weil RJ. Cerebral radiation necrosis: a review of the pathobiology, diagnosis and management considerations. J Clin Neurosci 2013;20:485-502. [PubMed: 23416129]

[29]. Cheung MC, Chan AS, Law SC, Chan JH, Tse VK. Impact of radionecrosis on cognitive dysfunction in patients after radiotherapy for nasopharyngeal carcinoma. Cancer 2003;97:201926. [PubMed: 12673733] 
All lesions
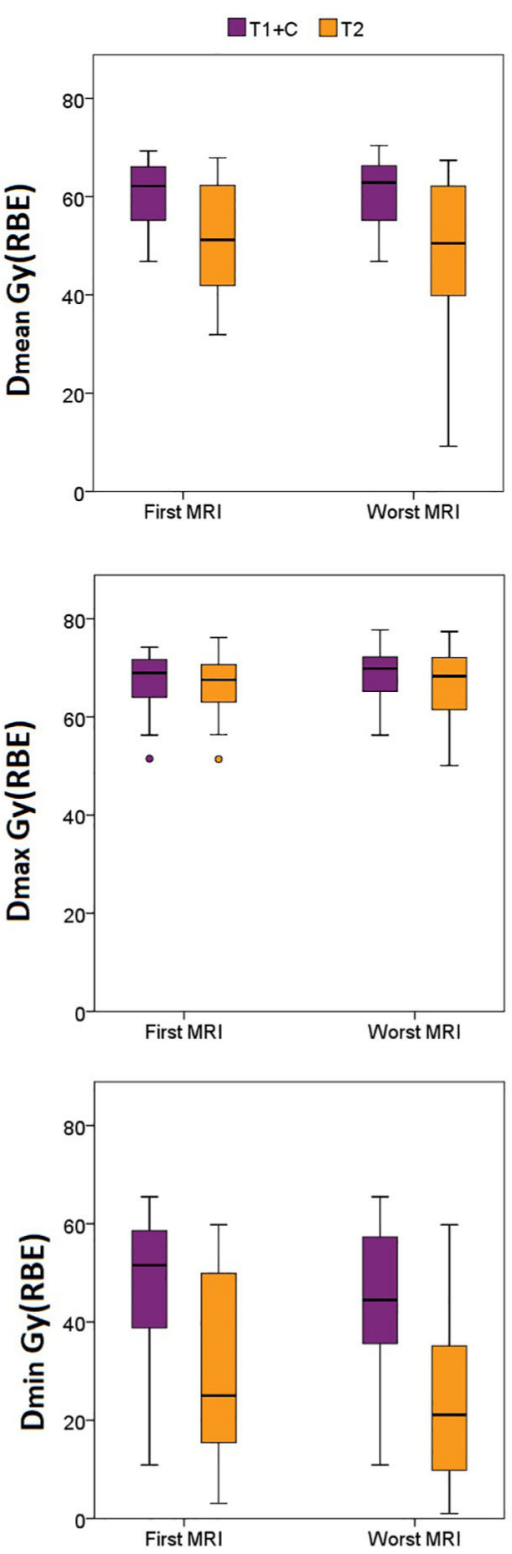

Brain location T1 lesions
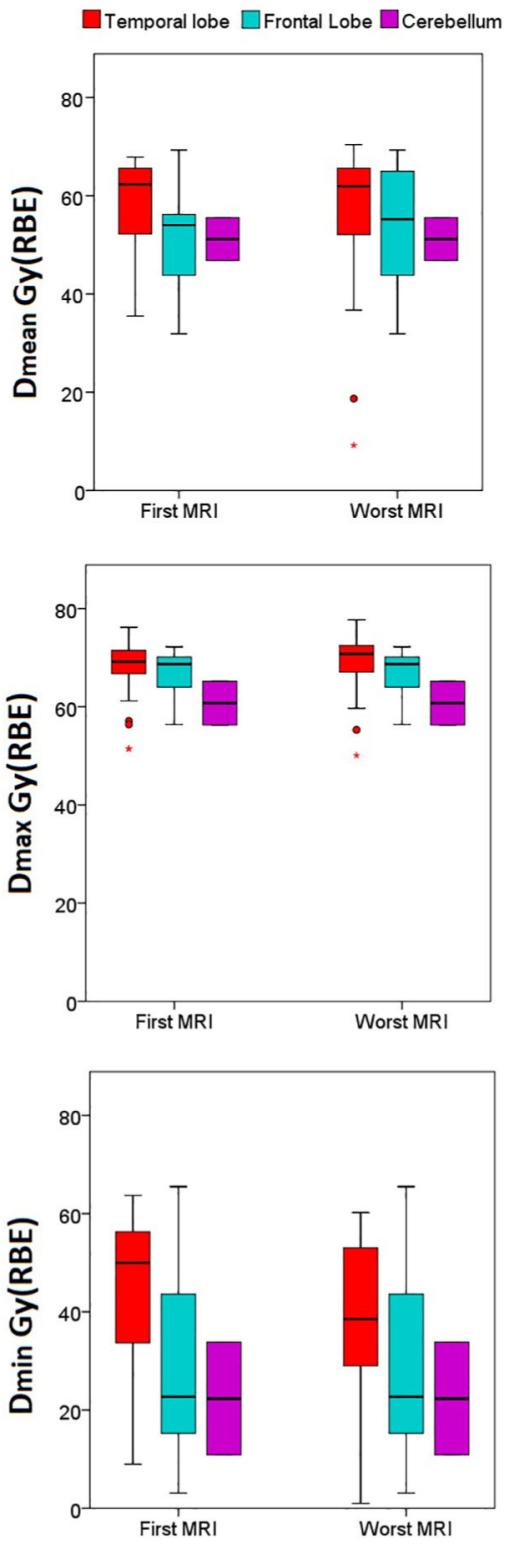

T1 lesions relative to CTV

$\square$ Outside $\square$ Overlapping $\square$ Inside
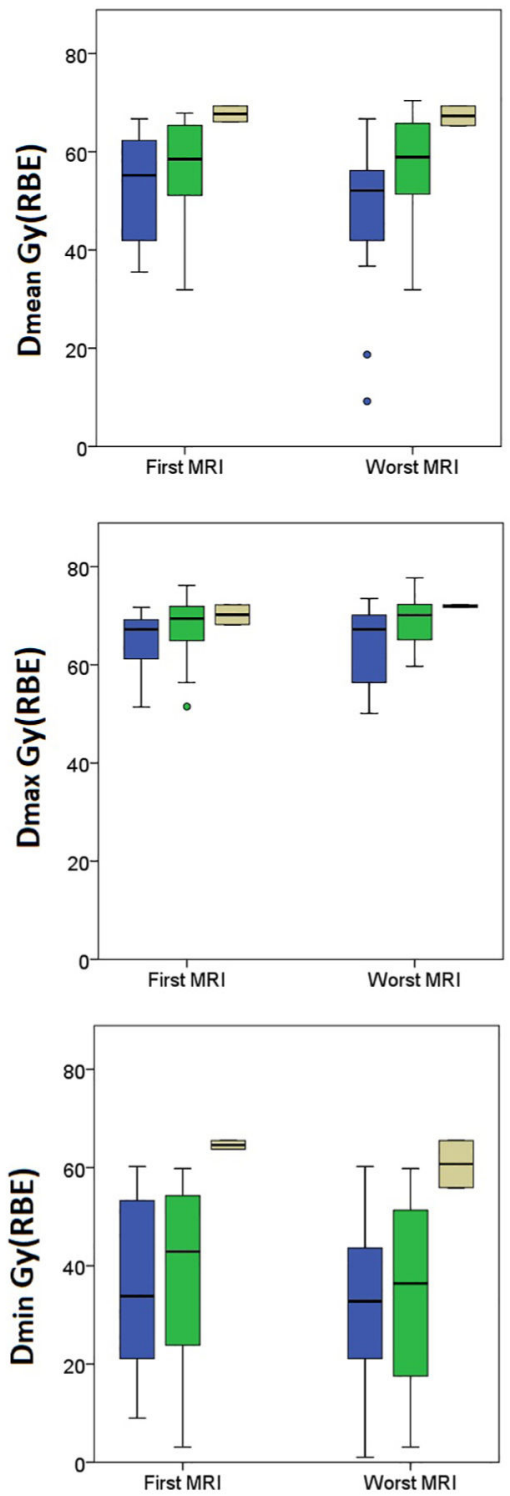

Fig. 1.

RAIC lesion doses for all lesions (left column), according to location in the brain (middle column) and location relative to the CTV (right column). 


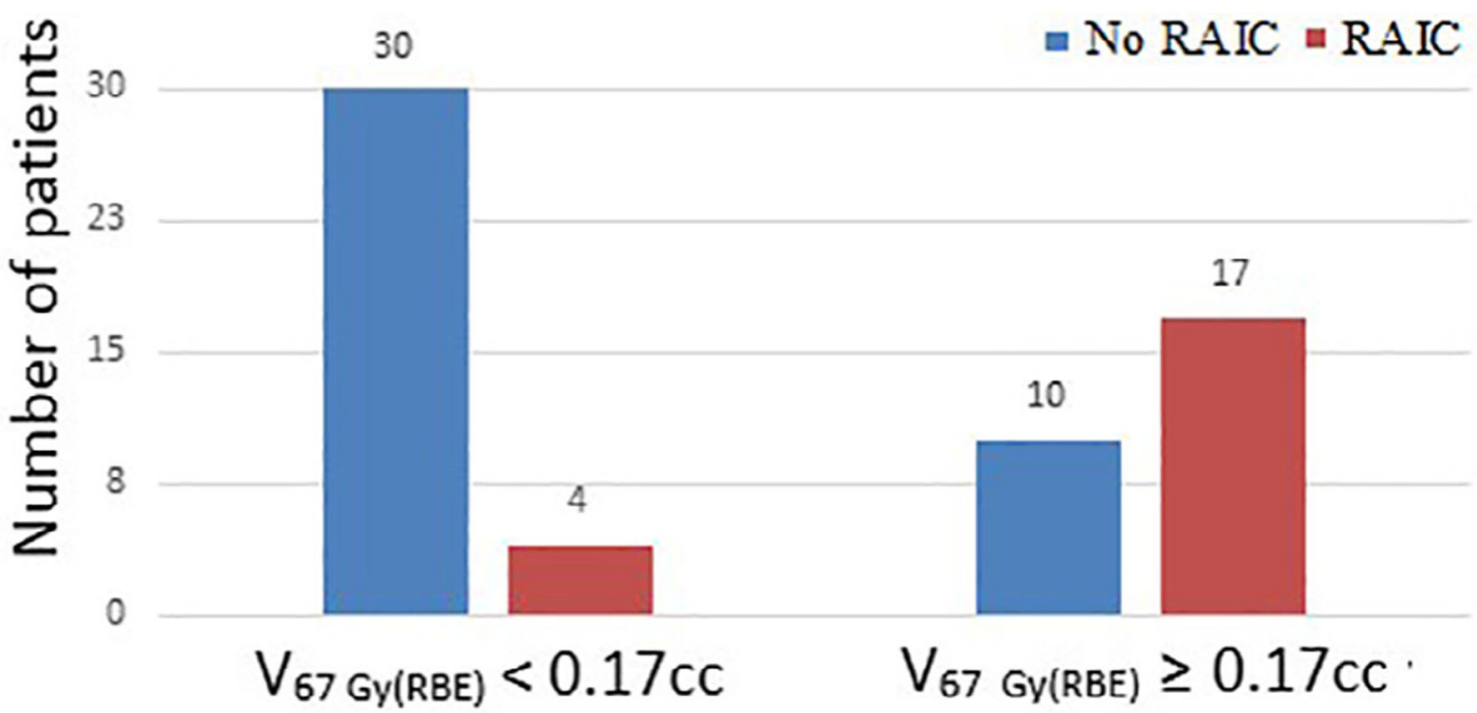

Fig. 2.

Results from RPA: 17 out of 27 patients with $V_{67} \mathrm{~Gy}(\mathrm{RBE}) \geq 0.17 \mathrm{cc}$ developed RAIC, whereas 4 out of 30 patients with $V_{67}$ Gy(RBE) $<0.17 \mathrm{cc}$ developed RAIC. 


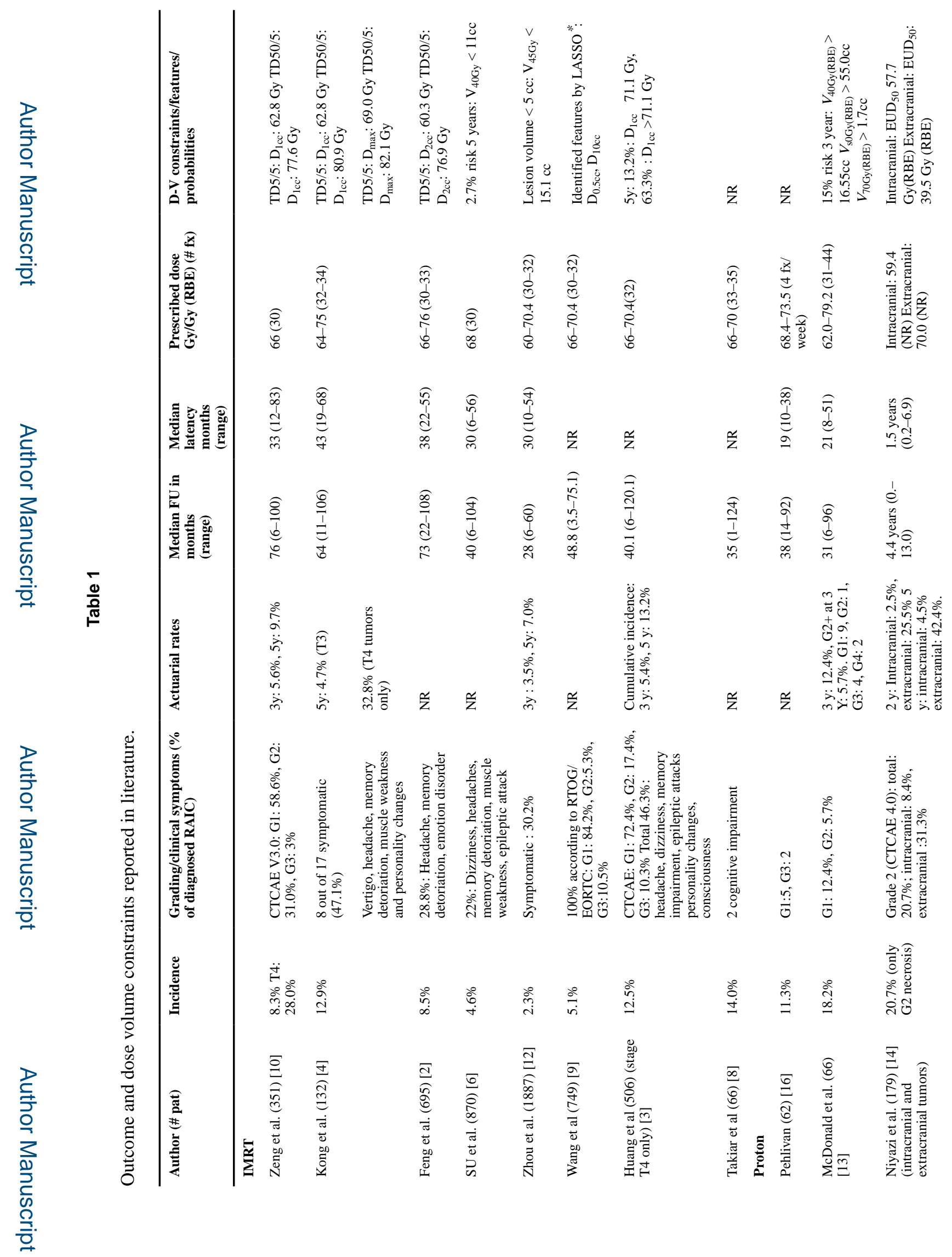

Radiother Oncol. Author manuscript; available in PMC 2021 October 01. 
Engeseth et al.

Page 13

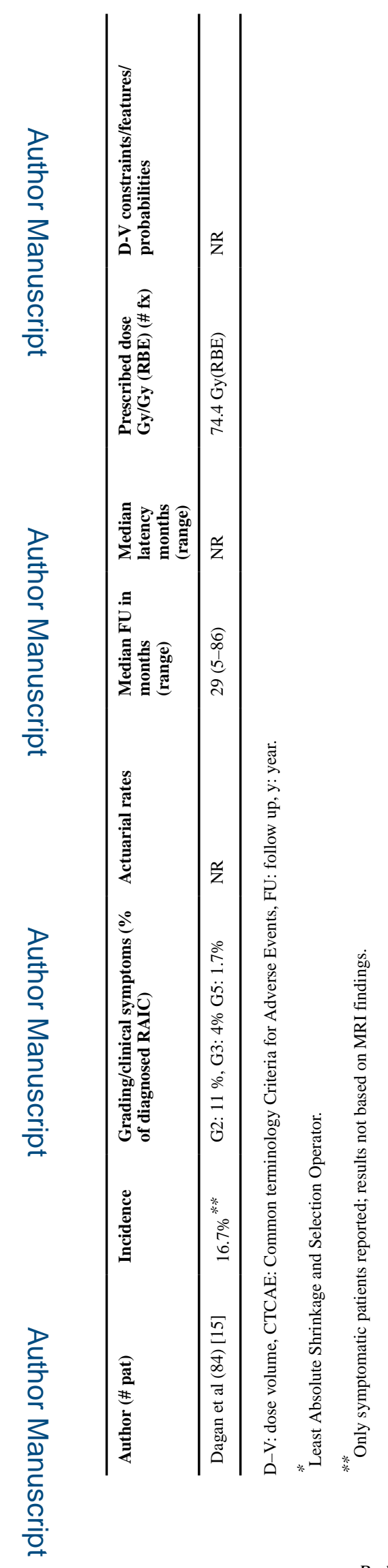

Radiother Oncol. Author manuscript; available in PMC 2021 October 01. 


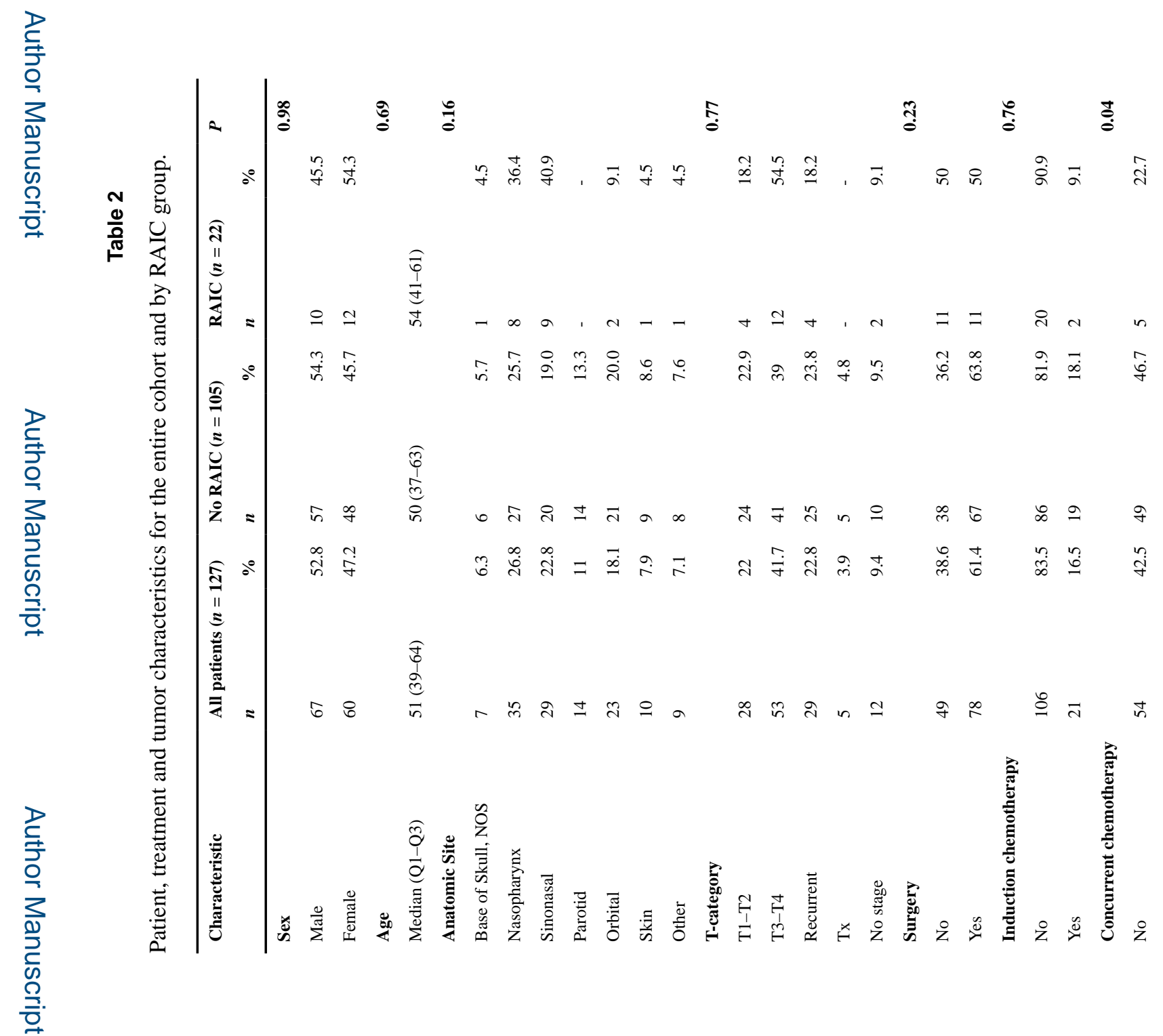

Radiother Oncol. Author manuscript; available in PMC 2021 October 01. 


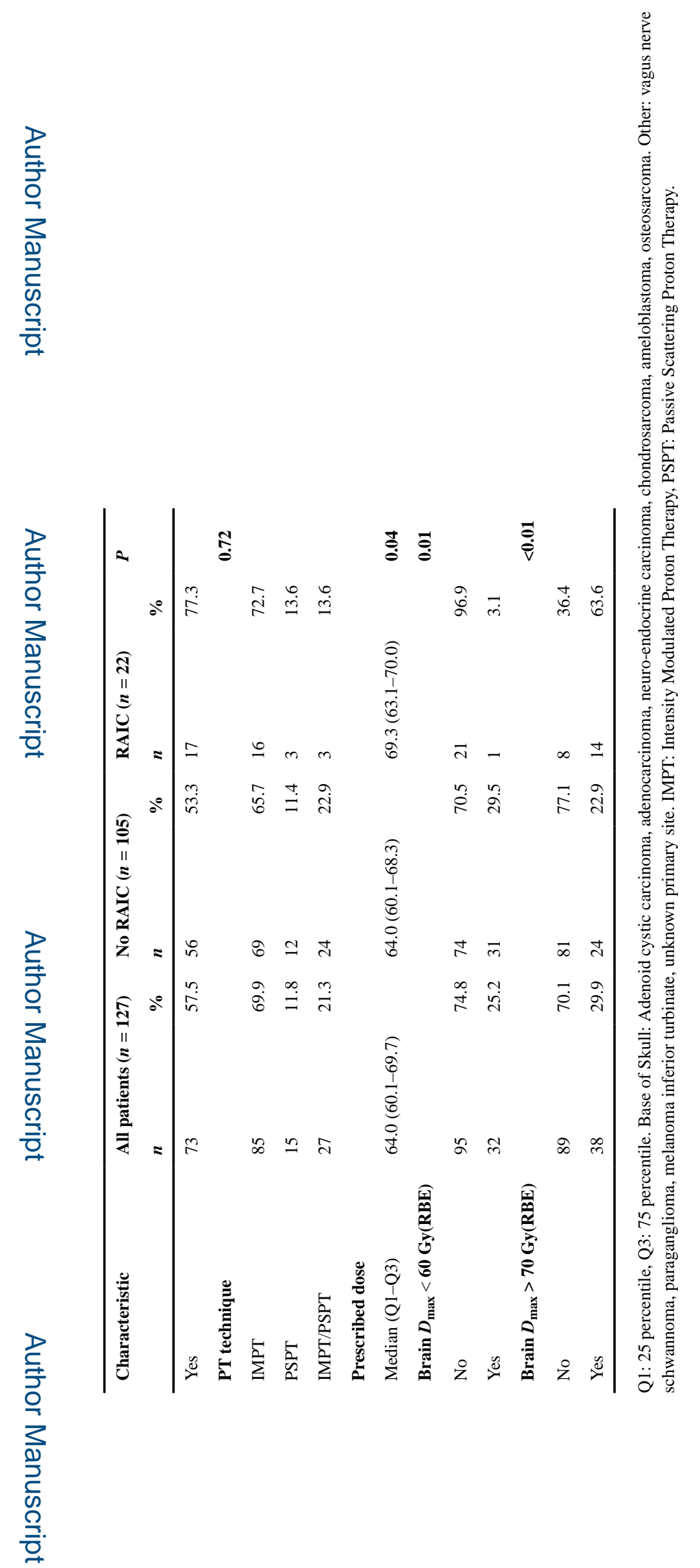

Radiother Oncol. Author manuscript; available in PMC 2021 October 01. 
Table 3

Dose volume statistics for those with and without RAIC. Median (Q1-Q3) reported.

\begin{tabular}{|c|c|c|c|}
\hline \multirow[b]{2}{*}{ Dose parameters brain } & No RAIC $(n=105)$ & RAIC $(n=22)$ & $P$ \\
\hline & \multicolumn{3}{|c|}{ Median Gy[RBE] (Q1-Q3) } \\
\hline$D_{\max }$ & $64.3(58.6-69.6)$ & $73.1(67.7-75.8)$ & $<.001$ \\
\hline$D_{1.0 \mathrm{cc}}$ & $59.1(51.4-64.7)$ & $67.0(62.8-72.1)$ & $<.001$ \\
\hline$D_{2.0 \mathrm{cc}}$ & $56.7(49.8-63.9)$ & $66.2(58.7-70.5)$ & $<.001$ \\
\hline$D_{3.0 \mathrm{cc}}$ & $54.3(48.2-64.4)$ & $64.7(57.9-69.0)$ & $<.001$ \\
\hline$D_{4.0 \mathrm{cc}}$ & $51.9(46.5-61.5)$ & $63.7(57.4-67.8)$ & $<.001$ \\
\hline$D_{5.0 \mathrm{cc}}$ & $50.3(45.3-60.7)$ & $63.0(56.3-66.7)$ & $<.001$ \\
\hline Volume parameters brain & \multicolumn{3}{|l|}{ Median cc (Q1-Q3) } \\
\hline$V_{40 \mathrm{~Gy}(\mathrm{RBE})}$ & $16.3(7.7-41.4)$ & $40.4(25.8-63.9)$ & 0.001 \\
\hline$V_{45 \mathrm{~Gy}(\mathrm{RBE})}$ & $10.0(5.2-31.5)$ & $30.2(17.3-49.9)$ & 0.001 \\
\hline$V_{50 \mathrm{~Gy}(\mathrm{RBE})}$ & $5.1(1.8-21.1)$ & $23.7(10.7-36.2)$ & $<.001$ \\
\hline$V_{55 \mathrm{~Gy}(\mathrm{RBE})}$ & $2.7(0.11-13.6)$ & $14.3(6.2-25.1)$ & $<.001$ \\
\hline$V_{60 \mathrm{~Gy}(\mathrm{RBE})}$ & $0.5(0-6.9)$ & $9.2(1.5-16.7)$ & 0.001 \\
\hline$V_{65} \mathrm{~Gy}(\mathrm{RBE})$ & $0.0(0.0-0.9)$ & $2.4(0.4-6.8)$ & $<.001$ \\
\hline$V_{70 \mathrm{~Gy}(\mathrm{RBE})}$ & $0.0(0.0-0.0)$ & $0.14(0.0-2.7)$ & $<.001$ \\
\hline
\end{tabular}




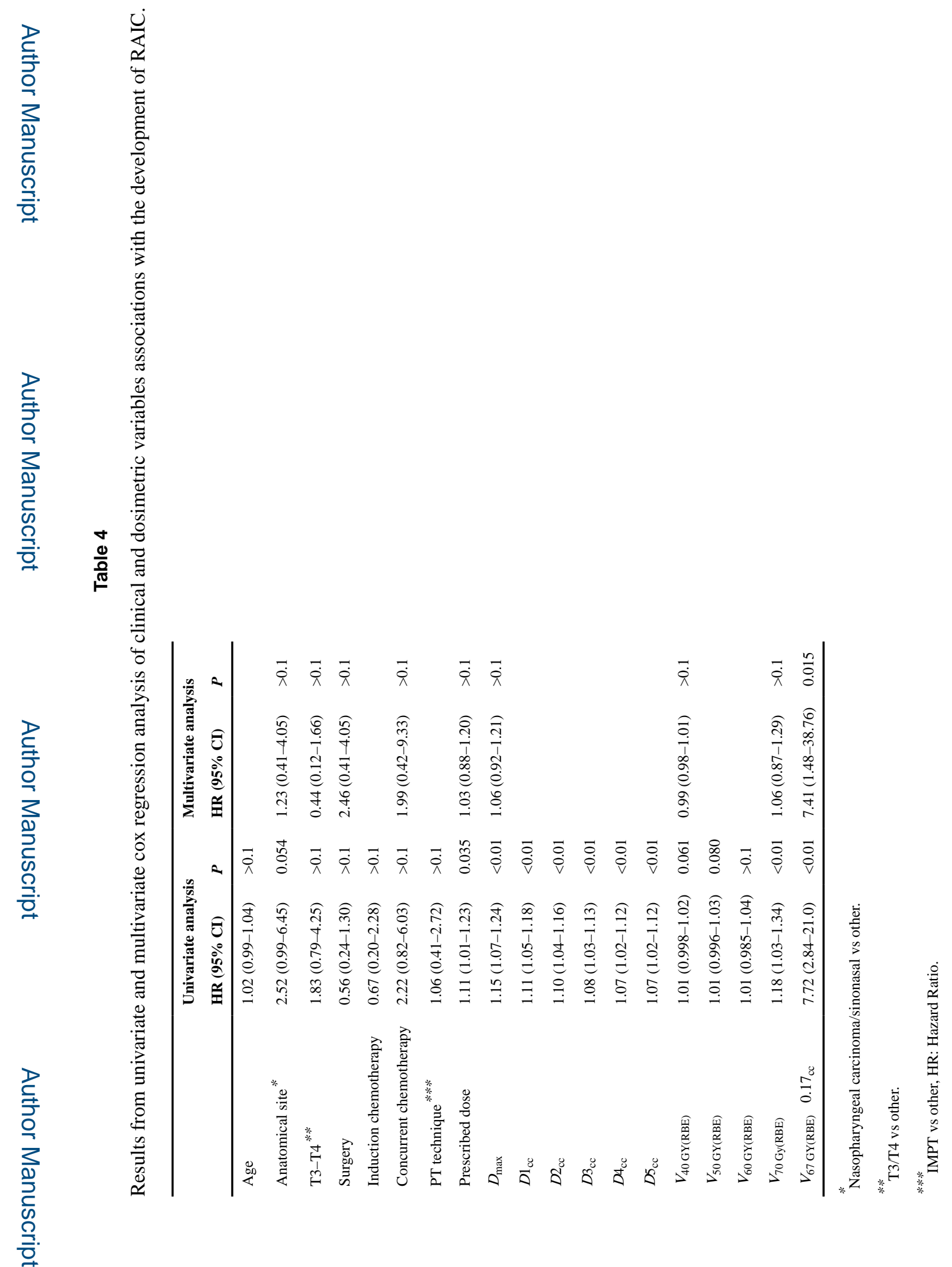

Radiother Oncol. Author manuscript; available in PMC 2021 October 01. 